\title{
Phonon-Assisted Electronic Transport through Double Quantum Dot System Coupled to Ferromagnetic Leads
}

\author{
P. TROCHA AND W. RUdZińsKI \\ Faculty of Physics, Adam Mickiewicz University, Umultowska 85, 61-614 Poznań, Poland
}

\begin{abstract}
Phonon-assisted electronic tunneling is studied through a double quantum dot coupled in parallel to ferromagnetic electrodes. The current-voltage characteristics for the system are derived within the nonequilibrium Green function technique based on equation of motion. It is found that additional phonon-induced resonance peaks appear in the spectral function on both sides of the main resonances corresponding to the quantum dot energy levels. It is shown that the "molecular-like" resonances are reproduced in the phonon side bands in the differential conductance. A significant phonon-induced enhancement of tunnel magnetoresistance as well as tunnel magnetoresistance oscillations are also predicted.
\end{abstract}

DOI: $10.12693 /$ APhysPolA.124.843

PACS: 72.25.Mk, 73.23.Hk, 73.63.Kv

\section{Introduction}

The polaronic transport through molecular double quantum dots (DQD) has been studied recently in a number of papers [1-3]. It has been found that an interplay between single-electron tunneling and the excitation of localized phonon modes gives rise to phonon side bands in density of states of the dot, to the Franck-Condon suppression of the linear conductance as well as to phonon-induced resonances in differential conductance. These effects have been already observed experimentally in tunneling junctions based on $C_{60}$ molecules, carbon nanotubes as well as in electron-phonon cavities with QDs embedded in a freestanding GaAs/AlGaAs membrane [4-6].

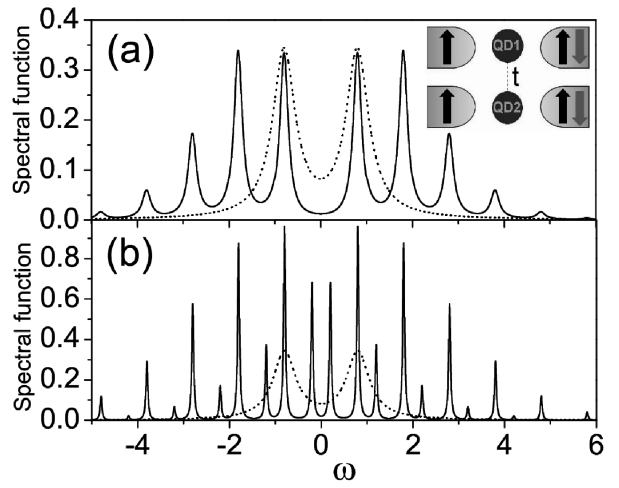

Fig. 1. Spectral function for spin-up electrons in $\mathrm{P}$ configuration versus $\omega$ calculated for thermal energies $k T=0$ (a) and $k T=1.2(\mathrm{~b})$. The other parameters are: $\lambda=1$ (solid lines), $\lambda=0$ (dotted lines), $\varepsilon^{\prime}=0, p=0.5$ and $\Gamma=0.2$. The inset shows the considered system: DQD $(t$ denotes the coupling strength between the dots QD1 and QD2) attached in parallel via tunneling barriers to external ferromagnetic electrodes. The arrows indicate that the magnetization of the right electrode may be aligned parallel or antiparallel with respect to the fixed magnetization of the left lead.

Up to now, the polaronic transport through DQDs has been studied theoretically only for non-magnetic tunnel- ing junctions. Thus, in this paper we investigate effects due to electron-phonon interaction in spin-polarized transmission through the system of DQD attached in parallel configuration to external ferromagnetic electrodes (see inset in Fig. 1). We examine the features of tunneling processes mediated through vibronic energy levels on DQD, behavior of the bonding and antibonding states in the presence of the phonon field as well as the influence of a local phonon mode on the tunnel magnetoresistance (TMR), which is due to a change in the junction resistance when magnetic moments of external electrodes are switched between the parallel $(\mathrm{P})$ alignment and the antiparallel (AP) one.

\section{Model and method}

Consider a parallel-coupled DQD attached via tunnel barriers to ferromagnetic leads, as shown in the inset of Fig. 1. The whole system can be described by Hamiltonian of the general form $H=H_{1}+H_{\mathrm{r}}+H_{\mathrm{ph}}+H_{\mathrm{DQD}}+H_{\mathrm{t}}$. The terms $H_{1}$ and $H_{\mathrm{r}}$ describe the left and right ferromagnetic electrodes, respectively, in the non-interacting quasi-particle approximation, $H_{\nu}=\sum_{k, \sigma} \varepsilon_{k \sigma} a_{k \sigma \nu}^{+} a_{k \sigma \nu}$, where $\varepsilon_{k \sigma}$ is the single-electron energy for wave vector $k$ and spin $\sigma(\sigma=\uparrow, \downarrow)$ whereas $a_{k \sigma \nu}^{+}$and $a_{k \sigma \nu}$ are the corresponding creation and annihilation operators for the $\nu$-th lead $(\nu=1, \mathrm{r})$. The third term is the phonon Hamiltonian, $H_{\mathrm{ph}}=\omega_{0} b^{+} b$, where $\omega_{0}$ is the vibrational frequency of the phonon mode and $b^{+}(b)$ is the phonon creation (annihilation) operator. The DQD system is described by $H_{\mathrm{DQD}}=\sum_{i \sigma}\left[\varepsilon_{i d}+\lambda\left(b+b^{+}\right)\right] d_{i \sigma}^{+} d_{i \sigma}-t \sum_{\sigma}\left(d_{1 \sigma}^{+} d_{2 \sigma}+\right.$ H.c.) $+\sum_{i} U_{i} n_{i \sigma} n_{i-\sigma}$, where $\varepsilon_{i d}$ denotes the energy of the discrete level of the $i$-th $\operatorname{dot}(i=1,2), d_{i \sigma}^{+}$and $d_{i \sigma}$ are the creation and annihilation operators for spin $\sigma$, $t$ denotes the interdot hopping parameter, $n_{i \sigma}=d_{i \sigma}^{+} d_{i \sigma}$ is the particle number operator, $U_{i}$ is the electron correlation parameter for two electrons residing on the $i$-th dot, while the parameter $\lambda$ describes strength of the electron-phonon coupling. Finally, the tunnelling term $H_{\mathrm{t}}$ describes processes due to coupling of DQD to the external leads, $H_{\mathrm{t}}=\sum_{k \nu} \sum_{i \sigma}\left(T_{i k \sigma}^{\nu} a_{k \sigma \nu}^{+} d_{i \sigma}+\right.$ H.c. $)$, where $T_{i k \sigma}^{\nu}$ are the relevant tunneling amplitudes. If the quan- 
tities $T_{i k \sigma}^{\nu}$ are real and constant, then coupling of the dots to external electrodes can be written as the $2 \times 2$ matrix $\boldsymbol{\Gamma}_{\sigma}^{\nu}$ with elements for the $\mathrm{P}$ configuration in the form $\Gamma_{i i \sigma}^{\mathrm{l}}=\beta^{\delta_{2 i}} \Gamma\left(1 \pm p_{\mathrm{l}}\right), \Gamma_{i i \sigma}^{\mathrm{r}}=\gamma \beta^{\delta_{1 i}} \Gamma\left(1 \pm p_{\mathrm{r}}\right)$, and $\Gamma_{i j \sigma}^{\nu}=\Gamma_{j i \sigma}^{\nu *}=q_{\nu} \gamma^{\delta_{\nu r}} \Gamma \sqrt{\beta}\left(1 \pm p_{\mathrm{r}}\right)$ with upper (lower) sign corresponding to $\sigma=\uparrow(\downarrow)$. The symbol $p_{\nu}$ denotes polarization of the $\nu$-th lead, $\Gamma$ is a constant, $\beta$ determines difference in the coupling of a given lead to the dots, $\gamma$ describes asymmetry in the coupling of the dots to the leads, whereas $q_{\nu}$ in general obey the condition $\left|q_{\nu}\right| \leq 1[7]$.

Using the Lang-Firsov-type unitary transformation [8], one may eliminate the linear coupling terms in the $H_{\mathrm{DQD}}$ Hamiltonian leading to renormalization of the dots energy levels $\varepsilon_{i d}^{\prime}=\varepsilon_{i d}-\lambda^{2} / \omega_{0}$ and charging energy $U_{i}^{\prime}=U_{i}-2 \lambda^{2} / \omega_{0}$. Assuming that the local polaron is localized, i.e. assuming that hopping is small compared to electron-phonon interactions, $T_{i k \sigma}^{\nu} \ll \lambda$, we adopt here the approximation developed for the independent boson model [8]. This approximation gives rise to exponential suppression of the tunneling amplitudes in the tunneling term $H_{\mathrm{t}}$, which in turn leads to the charge conserving $\lambda$-dependent Franck-Condon blockade of tunneling processes between the dot and an external electrode.

To calculate the occupation numbers and electric current for the system considered, we make use of the nonequilibrium Green function defined on the Keldysh contour [9]. The explicit expression for the Green functions can be obtained by using the equation of motion method. Having found the Green functions, one can calculate electric current flowing from the $\nu$-th lead to the $\operatorname{dot}[9], I_{\nu}=\frac{e}{\hbar} \sum_{\sigma} \int_{-\infty}^{+\infty} \frac{\mathrm{d} \omega}{2 \pi} \operatorname{Tr}\left(\boldsymbol{\Gamma}_{\sigma}^{\nu}\left(\boldsymbol{G}_{\sigma}^{<}(\omega)+\right.\right.$ $\left.f_{\nu}(\omega) \boldsymbol{A}_{\sigma}(\omega)\right)$ ), where $f_{\nu}(\omega)$ is the Fermi-Dirac distribution function, the quantity $\boldsymbol{A}_{\sigma}(\omega)$ stands for the spectral function, $\boldsymbol{A}_{\sigma}(\omega)=i\left[\boldsymbol{G}_{\sigma}^{>}(\omega)-\boldsymbol{G}_{\sigma}^{<}(\omega)\right]$ with $\boldsymbol{G}_{\sigma}^{<(>)}(\omega)$ being the Fourier transform of the lesser (greater) Green function of the dots. Consequently, the TMR characteristics may be evaluated from the ratio $\mathrm{TMR}=$ $\left(I_{\mathrm{P}}-I_{\mathrm{AP}}\right) / I_{\mathrm{AP}}$, where $I_{\mathrm{P}}$ and $I_{\mathrm{AP}}$ are electric tunneling currents in the $\mathrm{P}$ and $\mathrm{AP}$ magnetic configuration of the external electrodes, respectively.

\section{Numerical results}

In the following we shall discuss features of the transport characteristics of the system: the spectral function, differential conductance and TMR. For numerical analysis we assume equal dot levels, $\varepsilon_{1}^{\prime}=\varepsilon_{2}^{\prime} \equiv \varepsilon^{\prime}$. Moreover, for the on-dot charging energies we take $U_{1}^{\prime}=U_{2}^{\prime}=0$, i.e. it is assumed that effectively, for a given $\lambda$, the polaron shift gives rise to vanishing intradot Coulomb correlations [10]. The DQD is assumed to be coupled to magnetic electrodes with the equal polarizations, $p_{\mathrm{l}}=p_{\mathrm{r}} \equiv p$ and symmetrical DQD-lead couplings are taken into account with $\Gamma=0.2$ and $\gamma=1$. Apart from this, we consider the case of $q_{\nu}=0$, i.e. assume that each dot is effectively attached to its own pair of external electrodes, and for the asymmetry in the coupling of a given electrode to the dots we take $\beta=1$. Finally, the interdot hopping term is taken as $t=0.8$, which is (similarly as $\Gamma$ ) typical experimental value for a DQD setup (see e.g. [11]). All the energy parameters are measured relative to the phonon excitation frequency $\omega_{0}$.

The essential features of spectral functions for spin-up electrons tunneling in the $\mathrm{P}$ configuration, versus $\omega$ are shown in Fig. 1. In the system where electron-phonon interactions are negligible, $\lambda=0$, two resonance peaks (the dotted curves in Fig. 1) are visible. This is due to the fact that the interdot hopping term lifts the level degeneracy, thus giving rise to the so-called bonding and antibonding states, separated from each other in terms of energy by $2 t$. It is worth noting here that in our calculations we have observed a difference in height and width of the resonance peaks for up spin and down spin (not shown) orientations, which indicates that in the $\mathrm{P}$ configuration one should expect down spin electrons reside longer on the dot than up spin ones. Also, one would obtain for the AP configuration qualitatively the same picture as shown in Fig. 1, except that the spectral curves for opposite spin orientations would overlap. The latter follows from the fact that in symmetric junctions in the AP configuration the dot may be occupied by up spin and down spin electrons with the same probability.

When the electron-phonon interactions are switched on, the density of states is modified depending on the strength of the electron-phonon coupling as well as on the temperature. With increasing strength of the electronphonon coupling a polaron shift of the elastic resonances down in energy occurs. The displayed in Fig. 1 case of spectral function obtained for electron-phonon coupling $\lambda=1$ (solid line), which gives rise to polaron shift resulting in $\varepsilon^{\prime}=0$, shows that besides the main resonances also satellite sidebands spaced at the phonon energies appear. These satellite peaks are formed on the right and left side of the main resonances. Since at $k T=0$ there are no phonons to absorb, in Fig. 1a only peaks due to phonon emission are visible. With increasing temperature also the phonon absorption satellite peaks emerge in the density of states spectrum, which are clearly seen in Fig. 1b. Thermal fluctuations give rise to enhancement of the spectral function intensity thus increasing also the probability of tunneling via the phonon energy levels. The energies at which the phonon absorption resonances emerge are controlled by the strength of the interdot coupling. Similarly as the emission peaks, the absorption phonon satellites are also spaced at the phonon energy however, by contrast to the behavior of the emission sidebands, these are shifted towards lower energies relative to the main resonance as well as relative to each emission phonon resonance by $2 t-\omega_{0}$.

In Fig. 2a we show the behavior of the differential conductance as the function of the transport bias voltage. The dots levels are taken to be $\varepsilon_{1}=\varepsilon_{2}=1$, so that according to the condition for phonon-driven renormalization of the dots levels, for the assumed electronphonon coupling parameter $\lambda=1$, effectively the discrete levels experience the polaron shift giving rise to $\varepsilon^{\prime}=0$. For reference, the conductance in case of van- 


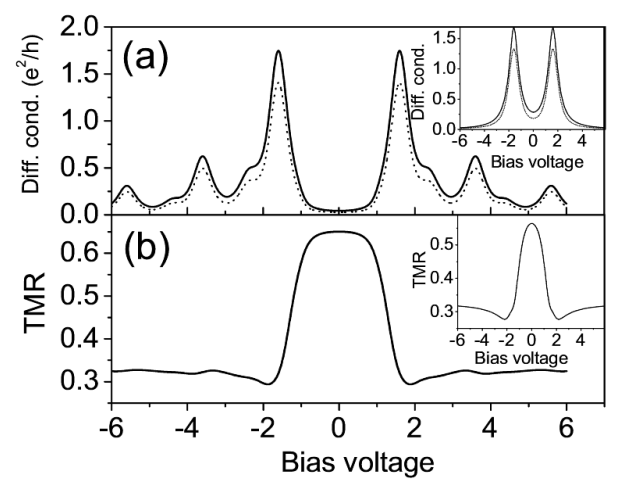

Fig. 2. Differential conductance (a) and TMR (b) calculated for $\lambda=1, \varepsilon^{\prime}=0, p=0.5, \Gamma=0.2$, and $k T=0.05$. Differential conductance is shown for up spin electrons in $\mathrm{P}$ (solid line) and AP (dotted line) configuration. The insets show the corresponding characteristics for $\lambda=0$.

ishing electron-phonon interactions, $\lambda=0$, is also displayed (see inset in Fig. 2a). As stated earlier, the position of the main resonances due to the tunneling through the bonding and antibonding states on the dot depends on the strength of the interdot coupling, and these appear at bias voltages $\pm 2 t$. If the temperature is set at $T=0$, then due to inelastic tunneling assisted by the phonon emission, additional steps in the current appear, and the phonon emission resonances in the differential conductance emerge. In the system based on DQD the phonon sidebands exhibit a double-peak structure. It is due to the fact that the phonon satellites form also the "molecular-like" states. The bias voltages at which these sidebands are formed may be readily evaluated from the oscillative behavior of the differential conductance displayed in Fig. 2a. First, notice that one series of phonon side resonances appear at bias voltages $2\left(t+n \omega_{0}\right)(n=1,2,3 \ldots)$. The latter result is analogous to the one observed earlier in tunneling junctions based on single QDs [12]. Second, the series of peaks due to tunneling through the other "molecular-like" vibrational states emerge at bias voltages $2\left[\omega_{0}(n+1)-t\right]$ $(n=1,2,3 \ldots)$. Figure $2 \mathrm{~b}$ shows the corresponding TMR quantity. It is seen that phonon-assisted tunneling gives rise to oscillations of TMR for transport occurring above the threshold bias voltages $\pm 2 t$. Also, in comparison to the case of $\lambda=0$ (see inset in Fig. 2b) the phonon-induced enhancement of TMR reaching $9 \%$ occurs in the bias voltage range where the sequential tunneling is exponentially suppressed. The latter indicates on the fact that phonon-assisted tunneling may increase the probability of higher order tunneling processes occurring below the first threshold bias voltage.

\section{Summary and conclusions}

Using the non-equilibrium Green function approach we have considered tunneling through a parallel-coupled DQD attached to ferromagnetic leads, in the presence of electron-phonon interactions. Phonon spectra calculated for different temperatures show that spin-polarized electron transmission may be mediated by the phonon energy levels. As in the systems based on a single molecular QDs, these tunneling processes are accompanied by renormalization of the dots energy levels as well as by appearance of vibrational mode resonances. On the other hand, it is found that for the DQD-based tunneling junction the emission vibronic sidebands are always spaced by the phonon energy from other nearest neighboring emission resonances as well as from the main resonance, while the position of the absorption peaks is always shifted relative to the emission ones, depending on the interdot coupling strength. It is found also that in nonequilibrium situation the phonon sidebands exhibit a double-peak structure, i.e. the bonding and antibonding states are reproduced in the satellite phonon peaks, and thus the corresponding TMR quantity experiences oscillations in the sequential tunneling regime. Also, by contrast to the case of tunneling junctions based on a single QD, a significant enhancement of the TMR maximum was predicted for bias voltages at which the sequential current is exponentially suppressed.

\section{Acknowledgments}

This work was supported by the Polish Ministry of Science and Higher Education as a research project in the years 2010-2013. P.T. also acknowledges support by the European Union under European Social Fund Operational Programme "Human Capital" (POKL.04.01.01-00133/09-00).

\section{References}

[1] X. Lu, J. Wang, C.-Q. Wu, Eur. Phys. J. B 49, 325 (2006).

[2] M. Bagheri Tagani, H. Rahimpour Soleimani, Phys. Scr. 86, 035706 (2012).

[3] C. Wang, J. Ren, B.W. Li, Q.H. Chen, Eur. Phys. J. B 85, 110 (2012).

[4] H. Park, J. Park, A.K.L. Lim, E.H. Anderson, A.P. Alivisatos, P.L. McEuen, Nature 407, 57 (2000).

[5] E.M. Weig, R.H. Blick, T. Brandes, J. Kirschbaum, W. Wegscheider, M. Bichler, J.P. Kotthaus, Phys. Rev. Lett. 92, 046804 (2004).

[6] R. Leturcq, C. Stampfer, K. Inderbitzin, L. Durrer, C. Hierold, E. Mariant, M.G. Schultz, F. von Oppen, K. Ensslin, Nat. Phys. 5, 327 (2009).

[7] P. Trocha, J. Barnaś, Phys. Rev. B 76, 165432 (2007).

[8] G.D. Mahan, Many-Particle Physics, Plenum Press, New York 2000.

[9] H. Haug, A.-P. Jauho, Quantum Kinetics in Transport and Optics of Semiconductors, Springer-Verlag, Berlin 1996.

[10] S.-N. Zhang, W. Pei, T.-F. Fang, Q.-F. Sun, Phys. Rev. B 86, 104513 (2012).

[11] F.J. Kaiser, S. Kohler, P. Hänggi, M. Malecha, J. Ebbecke, A. Wixforth, H. Schumacher, B. Kästner, D. Reuter, A.D. Wieck, J. Phys., Condens. Matter 20, 374108 (2008).

[12] W. Rudziński, J. Phys., Condens. Matter 20, 275214 (2008). 